\title{
Aging Effects on Behavioural Estimates of Suppression with Short Suppressors
}

\author{
Erica L. Hegland and Elizabeth A. Strickland
}

\begin{abstract}
Auditory two-tone suppression is a nearly instantaneous reduction in the response of the basilar membrane to a tone or noise when a second tone or noise is presented simultaneously. Previous behavioural studies provide conflicting evidence on whether suppression changes with increasing age, and aging effects may depend on whether a suppressor above (high-side) or below (low-side) the signal frequency is used. Most previous studies have measured suppression using stimuli long enough to elicit the medial olivocochlear reflex (MOCR), a soundelicited reflex that reduces cochlear amplification or gain. It has a "sluggish" onset of approximately $25 \mathrm{~ms}$. There is physiological evidence that suppression may be reduced or altered by elicitation of the MOCR. In the present study, suppression was measured behaviourally in younger adults and older adults using a forward-masking paradigm with 20-ms and 70-ms maskers and suppressors. In experiment 1, gain was estimated by comparing on-frequency $(2 \mathrm{kHz})$ and off-frequency $(1.2 \mathrm{kHz})$ masker thresholds for a short, fixed-level $2-\mathrm{kHz}$ signal. In experiment 2 , the fixedlevel signal was preceded by an off-frequency suppressor $(1.2$ or $2.4 \mathrm{kHz})$ presented simultaneously with the on-frequency masker. A suppressor level was chosen that did not produce any forward masking of the signal. Suppression was measured as the difference in on-frequency masker threshold with and without the suppressor present. The effects of age on gain and suppression estimates will be discussed.
\end{abstract}

Keywords Two-tone suppression - Medial olivocochlear reflex - MOCR • Endocochlear potential $\cdot$ Gain estimates $\cdot$ Duration

\section{Introduction}

We live in a noisy world, and understanding speech in noise is a common challenge. As people age, this task becomes increasingly difficult, and elevated thresholds alone cannot account for this increased difficulty (e.g., Pichora-Fuller et al. 1995). One explanation for this discrepancy may be attributed to the decrease in the en-

\footnotetext{
E. L. Hegland $(\bowtie) \cdot$ E. A. Strickland

Purdue University, 715 Clinic Drive, West Lafayette, IN 47907, USA

e-mail: ehegland@purdue.edu 
docochlear potential that tends to occur with increasing age (e.g., Schuknecht et al. 1974). A decreased endocochlear potential negatively affects the function of inner and outer hair cells (OHCs) (e.g., Gates et al. 2002), and disruptions in OHC function could decrease cochlear nonlinearity (e.g., Schmiedt et al. 1980).

A sensitive measure of cochlear nonlinearity is two-tone suppression, e.g., a nearly instantaneous reduction in the basilar membrane (BM) response to one tone in the presence of a second tone. There is psychoacoustic evidence that suppression measured with suppressors above the signal frequency (high-side suppression) and below the signal frequency (low-side suppression) may show different aging effects. Several of these psychoacoustic studies have used a band-widening technique in a forward-masking paradigm to measure suppression (Dubno and Ahlstrom 2001a, b; Gifford and Bacon 2005). Narrow bandpass noise centred at the signal frequency was widened by adding noise bands above, below, or above and below the signal frequency. Suppression was measured as the difference in signal threshold between the narrowest and widest noisebands. Dubno and Ahlstrom (2001a, b) found less suppression with more noise above rather than below the signal frequency, and the older adults with normal hearing had less suppression than the younger adults. Older adults had minimal low-side suppression and absent high-side suppression (Dubno and Ahlstrom 2001a). In contrast, using the band-widening technique with noise added below the signal, Gifford and Bacon (2005) found no effect of age on suppression. Using tonal maskers and suppressors in a forward-masking paradigm, Sommers and Gehr (2010) found reduced or absent high-side suppression with increased age.

It is possible that previous psychoacoustic studies of suppression underestimated the amount of suppression by using maskers that were long enough to elicit the medial olivocochlear reflex (MOCR). The MOCR is a sound-elicited efferent feedback loop that reduces OHC amplification or gain (Cooper and Guinan 2006). Gain starts to be affected approximately $25 \mathrm{~ms}$ after elicitor onset. Evidence from physiological studies in animals suggests that elicitation of the MOCR may decrease or modify suppression (Winslow and Sachs 1987; Kawase et al. 1993). Previous studies of aging effects on suppression used masker and suppressor durations of 200-500 ms (Dubno and Ahlstrom 2001a, b; Gifford and Bacon 2005; Sommers and Gehr 1998, 2010). These durations were long enough to elicit MOCR-induced gain reductions and possibly reduce suppression estimates.

The goal of this study was to investigate the effect of age on estimates of twotone suppression using shorter stimuli. Several prior studies of aging effects on suppression have used masker levels that had been previously shown to produce large amounts of suppression in young adults (Dubno and Ahlstrom 2001a, 2001b; Gifford and Bacon 2005; Sommers and Gehr 2010). In the present study, suppressor levels were chosen that did not produce forward masking of the signal, which can be presumed to produce minimal spread of excitation at the signal frequency place (Moore and Vickers 1997). If simultaneous masking is produced by spread of excitation and suppression, using a suppressor that does not produce forward masking should result in a measure of suppression rather than a mixture of suppression and excitation. 


\section{Methods}

\subsection{Participants}

Participants included five younger adults between 18 and 30 years old ( mean $=21.4$; 3 female) and five older adults between 62 and 70 years old (mean $=65.4 ; 4$ female). The average threshold for younger adults at $2 \mathrm{kHz}$ was $5 \mathrm{~dB} \mathrm{HL}$ (SD 3.54), and the average threshold for older adults at $2 \mathrm{kHz}$ was $15 \mathrm{~dB}$ HL (SD 9.35). The Montreal Cognitive Assessment (MoCA; used with permission, copyright Z. Nasreddine) was administered, and all results were within the normal range $(\geq 26 / 30)$.

\subsection{Stimuli}

The signal was a $10-\mathrm{ms}, 2-\mathrm{kHz}$ sinusoid with $5-\mathrm{ms}$ onset and offset ramps and no steady state portion. This signal frequency has been used in previous studies on aging effects on suppression (Sommers and Gehr 1998, 2010; Dubno and Ahlstrom 2001a, 2001b; Gifford and Bacon 2005). The signal was presented at $10 \mathrm{~dB}$ SL and/or $50 \mathrm{~dB}$ SPL for each participant, and the masker level was adaptively varied to find threshold. Masker durations were 20 (short) and $70 \mathrm{~ms}$ (long). The $20-\mathrm{ms}$ masker should be too short to elicit the MOCR, while the 70-ms masker should be long enough to elicit full gain reduction from MOCR elicitation (Roverud and Strickland 2010). For experiment 1, masker thresholds for the short and long onfrequency $(2 \mathrm{kHz})$ and off-frequency $(1.2$ and $2.4 \mathrm{kHz})$ maskers were measured (Fig. 1). At the signal frequency place, the BM response to the 1.2-kHz masker should be approximately linear, even with MOCR stimulation (Cooper and Guinan 2006). The 2.4-kHz masker frequency was chosen because it has produced substantial suppression of a $2-\mathrm{kHz}$ masker in previous studies with young adults (Shannon 1976; Sommers and Gehr 2010). Gain was estimated as the difference in threshold between the on-frequency masker and the 1.2-kHz masker (Yasin et al. 2014). For experiment 2, suppression was measured by presenting a short or long, 1.2- or $2.4-\mathrm{kHz}$ suppressor simultaneously with the on-frequency masker and just
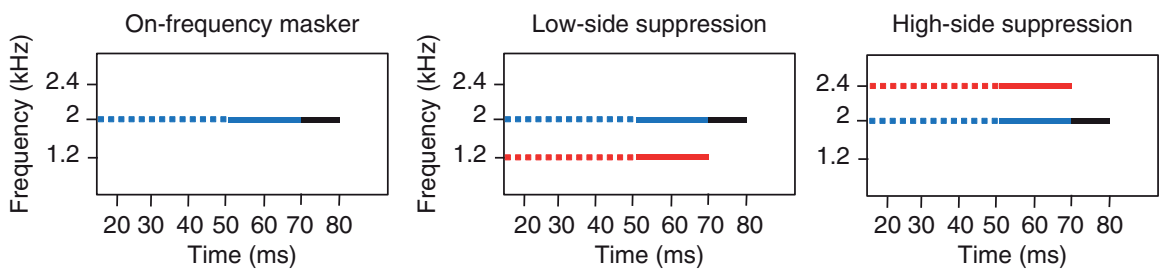

Fig. 1 Schematic of stimuli frequencies $(\mathrm{kHz})$ and durations $(\mathrm{ms})$. Masker durations were $20 \mathrm{~ms}$ (solid lines) and $70 \mathrm{~ms}$ (solid + dashed lines). Maskers were on-frequency (blue, $2 \mathrm{kHz}$ ) and offfrequency (red, 1.2 and $2.4 \mathrm{kHz})$ 
prior to presentation of the $10-\mathrm{ms}, 2-\mathrm{kHz}$ signal. This will be referred to as the "combined masker." The masker and suppressor durations were always equal. The on-frequency masker level was adaptively varied to find threshold.

All stimuli were digitally produced with MATLAB ${ }^{\mathrm{TM}}$ software (2012a, The Math Works, Natick, MA). They were output via a Lynx II XLR sound card to a headphone buffer (TDT HB6) and presented to the right ear or the better ear of each participant through an ER-2 insert earphone (Etymotic Research, Inc., Elk Grove Village, IL).

\subsection{Procedure}

Participants sat in a sound-attenuating booth and listed to stimuli presented to their right ear or better ear. Masker threshold for a fixed signal level was measured using a three-interval forced-choice adaptive procedure. Participants were instructed to use a computer mouse or keyboard to choose the interval that sounded different from the others. The masker level was increased following one incorrect response and decreased following two correct responses. Following this procedure, an estimate of $70 \%$ correct on the psychometric function is obtained (Levitt 1971). Masker threshold for a given trial was calculated as an average of the last eight of the 12 total reversals. Thresholds with a standard deviation of $5 \mathrm{~dB}$ or greater were not included in the final average. The order of the conditions was counterbalanced across the participants.

\section{Results}

\subsection{Experiment 1}

Thresholds for the short and long on-frequency $(2 \mathrm{kHz})$ and off-frequency maskers $(1.2$ and $2.4 \mathrm{kHz})$ were obtained for signal levels fixed at $10 \mathrm{~dB}$ above each participant's threshold for the 2-kHz, 10-ms tone. Because the absolute signal level differed between younger and older participants, masker thresholds were also obtained for a 50-dB SPL signal, which was 10-dB SL for some older participants.

Estimates of cochlear gain were calculated as the difference in masker threshold between the $2-\mathrm{kHz}$ and $1.2-\mathrm{kHz}$ short maskers (Yasin et al 2014). Gain was determined separately for each signal level. A one-way ANOVA was conducted to compare the effect of age group on gain estimates at each signal level. There was a significant effect of age group on gain estimates at the 10-dB SL signal level $[F(1,8)=7.03, p=0.029]$ and the 50-dB SPL signal level $[F(1,7)=6.86, p=0.034]$. Average amounts of gain are shown in Fig. 2. These results suggest that gain estimates were significantly decreased in older adults compared to younger adults. 
Fig. 2 Average amounts of gain for older and younger listeners at the $10-\mathrm{dB}$ SL and 50-dB SPL signal levels

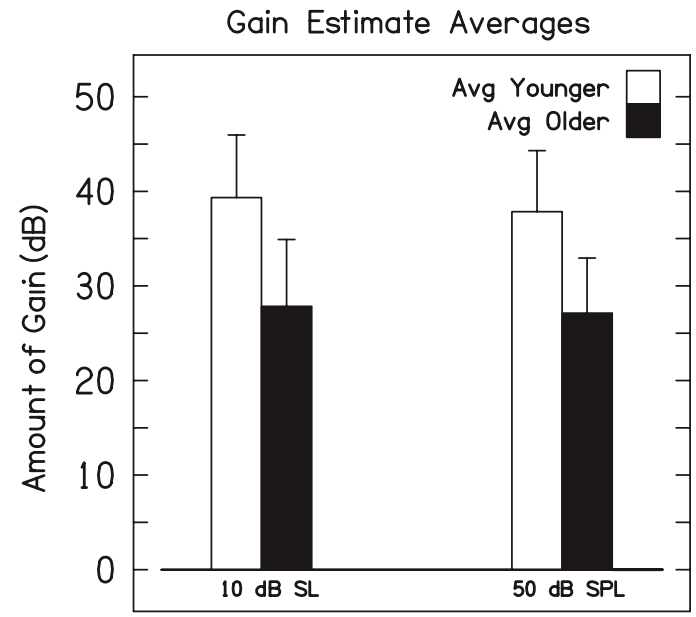

Correlation coefficients were computed between auditory threshold at $2 \mathrm{kHz}$ and gain estimates at both signal levels, and neither of the correlations reached statistical significance.

\subsection{Experiment 2}

Suppression was measured in a forward-masking paradigm (see Fig. 1). Suppressor levels were chosen that were below the thresholds obtained in Experiment 1 to ensure that the suppressor did not produce any forward masking of the signal. For each participant, several suppressor levels were presented, and the level producing the greatest amount of suppression was used for analysis. The suppressor was an average of $4 \mathrm{~dB}$ below the off-frequency masker threshold obtained in Experiment $1[S D=1.60]$. For younger adults, suppressors were presented at an average of $74.50 \mathrm{~dB}$ SPL $[S D=5.22]$ for low-side suppression and $74.23 \mathrm{~dB}$ SPL $[S D=4.00]$ for high-side suppression. For older adults, suppressors were presented at an average of $67.60 \mathrm{~dB}$ SPL [SD $=2.12]$ for low-side suppression and $75.20 \mathrm{~dB}$ $\mathrm{SPL}[S D=3.68]$ for high-side suppression. Compared to the $10 \mathrm{~dB}$ SL signal level, suppressors were on average $6.8 \mathrm{~dB}$ higher at the $50 \mathrm{~dB}$ SPL signal level for the younger adults and $2.7 \mathrm{~dB}$ higher for the older adults.

Average amounts of low-side and high-side suppression for younger adults (open bars) and older adults (filled bars) at the 10-dB SL and 50-dB SPL signal levels are shown in Fig. 3. With the short combined masker, all participants showed some low-side suppression, and all participants except for one older adult showed high-side suppression. With the long combined masker, $2 / 5$ older adults had no measurable low-side suppression, and 3/5 older adults had no measurable high-side suppression. A one-way ANOVA was conducted to compare the effect of age group 


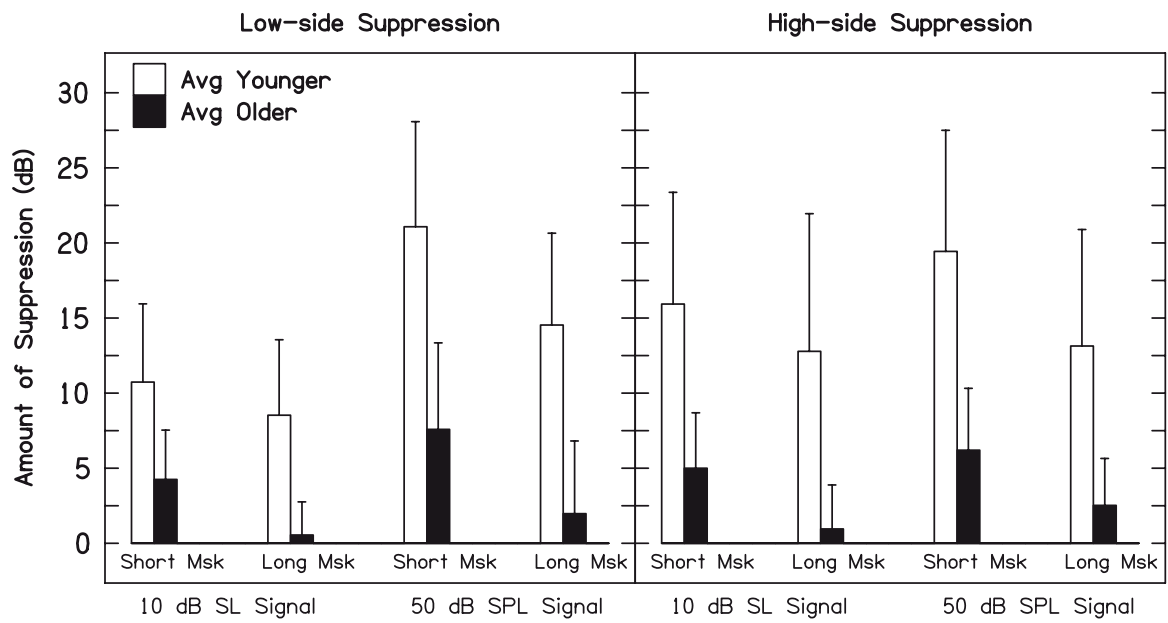

Fig. 3 Average suppression estimates for younger adults (open bars) and older adults (filled bars). Results are shown for the $1.2 \mathrm{kHz}$ suppressor (low-side suppression), $2.4 \mathrm{kHz}$ suppressor (high-side suppression), 20-ms combined masker (short msk), 70-ms combined masker (long $m s k), 10-\mathrm{dB}$ SL signal level, and 50-dB SPL signal level

on suppression estimates. There was a significant effect of age group on suppression estimates at the $p<0.05$ level for all conditions tested. There was a significant effect of age group for low-side suppression with a short combined masker [10-dB SL signal: $F(1,8)=5.53, p=0.047 ; 50-\mathrm{dB}$ SPL signal: $F(1,8)=11.09, p=0.010]$ and with a long combined masker [10-dB SL signal: $F(1,8)=10.57, p=0.012 ; 50-\mathrm{dB}$ SPL signal: $F(1,8)=12.94, p=0.007]$. There was a significant effect of age group for highside suppression with a short combined masker [10-dB SL signal: $F(1,8)=8.66$, $p=0.019 ; 50-\mathrm{dB}$ SPL signal: $F(1,7)=10.37, p=0.015]$ and with a long combined masker [10-dB SL signal: $F(1,8)=7.55, p=0.025 ; 50-d B$ SL signal: $F(1,7)=7.99$, $p=0.026]$. This suggests that both high-side and low-side suppression estimates decreased with age.

A paired-samples t-test was conducted to compare suppression estimates obtained with short and long combined maskers. In all suppression conditions tested, there were significant differences at the $p<0.05$ level between suppression estimates obtained with short and long combined maskers. There was a significant difference between low-side suppression estimates obtained with short $[M=7.49, S D=5.34]$ and long combined maskers $[M=4.54, S D=5.57]$ at the $10-\mathrm{dB}$ SL signal level $[t(9)=2.94, p=0.017]$ and between short $[M=14.33, S D=9.33]$ and long combined maskers $[M=8.25, S D=8.42]$ at the $50-\mathrm{dB}$ SPL signal level $[t(9)=3.41, p=0.008]$. There was a significant difference between high-side suppression estimates obtained with short $[M=10.46, S D=7.99]$ and long combined maskers $[M=6.86$, $S D=8.95]$ at the $10-\mathrm{dB}$ SL signal level $[t(9)=3.83, p=0.004]$ and between short $[M=12.08, S D=9.03]$ and long combined maskers $[M=7.24, S D=7.66]$ at the 50dB SPL signal level $[t(9)=4.56, p=0.002]$. This suggests that suppression estimates were significantly greater when measured with a short combined masker. 
It is possible that the decreased suppression in the older adults was due to their increased auditory thresholds. To determine the effect of auditory threshold on suppression estimates, correlation coefficients were computed between threshold in $\mathrm{dB}$ HL and suppression estimates from each of the eight conditions tested. Out of the eight suppression conditions, threshold at $2 \mathrm{kHz}$ was negatively correlated with low-side suppression at the 50-dB SPL signal level measured with the short combined masker $[r(10)=-0.68, p=0.031]$ and with the long combined masker $[r(10)=-0.67, p=0.33]$. This suggests that those with higher auditory thresholds had smaller low-side suppression estimates at the 50-dB SPL signal level. Otherwise, auditory threshold was not significantly correlated with either suppression or gain estimates.

\section{Discussion}

In the present experiment, several important differences were seen between younger adults and older adults. In Experiment 1, older adults were found to have less gain than younger adults, measured as the difference in on-frequency and off-frequency masker threshold for a fixed signal level (Yasin et al. 2014). The off-frequency, $1.2-\mathrm{kHz}$ masker is understood to have a linear BM response at the signal frequency place, and the on-frequency, 2-kHz masker and signal have a compressive BM response at the signal frequency place (Oxenham and Plack 2000). Comparing these two conditions gives an estimate of cochlear gain around the signal frequency place. It is possible that the decrease in gain for the older adults was due to their slightly poorer auditory thresholds. However, correlation coefficients between auditory threshold and gain estimates did not reach significance. This measure of gain may reflect decreases in cochlear function that occur prior to elevations in auditory thresholds. Decreased endocochlear potential may have less of an effect on thresholds closer to the apex of the cochlea and a larger effect in the base (Mills et al. 2006) while still impacting cochlear nonlinearity.

Estimates of high-side and low-side suppression were significantly smaller for older adults than for younger adults. Most previous studies of aging effects on suppression have found decreased or absent suppression for older adults (Sommers and Gehr 1998, 2010; Dubno and Ahlstrom 2001a, b). In the present study, suppressor levels were chosen to not produce forward masking and to result in the greatest measure of suppression. Also, with the long combined masker, some older adults did not show high-side suppression, and some did not show low-side suppression. By using short combined maskers with individually-selected suppressor levels, accurate measurements of suppression can be achieved.

Both younger and older adults in the present study showed significantly more suppression measured with short combined maskers than with longer combined maskers. Several of the older participants, who had no measurable suppression with the longer combined masker, had a small amount of suppression measured with the shorter combined masker. When suppression is measured with combined maskers 
long enough to elicit MOCR-induced gain reduction, suppression may be underestimated. However, even when measured with the shorter combined maskers, older adults had less suppression than younger adults. Evidence from animal studies suggests that gain reduction from the MOCR and suppression interact, resulting in decreased suppression (Winslow and Sachs 1987; Kawase et al. 1993). The results of the present study support this idea.

Dubno and Ahlstrom (2001a) found a negative correlation between auditory thresholds and suppression estimates. In the present study, there was a negative correlation between auditory thresholds and suppression estimates for low-side suppression at the 50-dB SPL signal level. The correlation was not significant for low-side suppression at the 10-dB SL signal level or for high-side suppression. The suppression estimates in Dubno and Ahlstrom (2001a) were measured between the narrowest and widest bandwidth maskers. This measure would include elements of both high-side and low-side suppression. It is possible that auditory threshold is more strongly correlated with low-side suppression.

The present study found strong evidence for age-related decreases in both highside and low-side two-tone suppression and in estimates of cochlear gain. These findings are consistent with a decreasing endocochlear potential that occurs with increasing age. The results also support the hypothesis that that suppression estimates may be underestimated when measured with long combined maskers, which would be consistent with a reduction in suppression due to the MOCR.

Acknowledgments This research was supported by a fellowship through NIH(NIDCD) T32 DC000030-21 and NIH(NIDCD) F31 DC014395 (first author), and NIH(NIDCD) R01 DC008327 (second author).

Open Access This chapter is distributed under the terms of the Creative Commons AttributionNoncommercial 2.5 License (http://creativecommons.org/licenses/by-nc/2.5/) which permits any noncommercial use, distribution, and reproduction in any medium, provided the original author(s) and source are credited.

The images or other third party material in this chapter are included in the work's Creative Commons license, unless indicated otherwise in the credit line; if such material is not included in the work's Creative Commons license and the respective action is not permitted by statutory regulation, users will need to obtain permission from the license holder to duplicate, adapt or reproduce the material.

\section{References}

Cooper NP, Guinan JJ Jr (2006) Efferent-mediated control of basilar membrane motion. J Physiol 576(1):49-54

Dubno JR, Ahlstrom JB (2001a) Psychophysical suppression effects for tonal and speech signals. J Acoust Soc Am 110(4):2108-2119

Dubno JR, Ahlstrom JB (2001b) Psychophysical suppression measured with bandlimited noise extended below and/or above the signal: effects of age and hearing loss. J Acoust Soc Am 110(2):1058-1066

Gates GA, Mills D, Nam B, D'Agostino R, Rubel EW (2002) Effects of age on the distortion product otoacoustic emission growth functions. Hear Res 163(1-2):53-60 
Gifford RH, Bacon SP (2005) Psychophysical estimates of nonlinear cochlear processing in younger and older listeners. J Acoust Soc Am 118(6):3823-3833

Kawase T, Delgutte B, Liberman MC (1993) Antimasking effects of the olivocochlear reflex. II. Enhancement of auditory-nerve response to masked tones. J Neurophysiol 70(6):2533-2549

Levitt H (1971) Transformed up-down methods in psychoacoustics. J Acoust Soc Am 49(2):467477

Mills JH, Schmiedt RA, Schulte BA, Dubno JR (2006) Age-related hearing loss: a loss of voltage, not hair cells. Semin Hear 27(4):228-236

Moore BCJ, Vickers DA (1997) The role of spread of excitation and suppression in simultaneous masking. J Acoust Soc Am 102(4):2284-2290

Oxenham AJ, Plack CJ (2000) Effects of masker frequency and duration in forward masking: further evidence for the influence of peripheral nonlinearity. Hear Res 150:258-266

Pichora- Fuller MK, Schneider BA, Daneman M (1995) How young and old adults listen to and remember speech in noise. J Acoust Soc Am 97(1):593-608

Roverud E, Strickland EA (2010) The time course of cochlear gain reduction measured using a more efficient psychophysical technique. J Acoust Soc Am 128(3):1203-1214

Schmiedt RA, Zwislocki JJ, Hamernik RP (1980) Effects of hair cell lesions on responses of cochlear nerve fibers. I. Lesions, tuning curves, two-tone inhibition, and responses to trapezoidalwave patterns. J Neurophysiol 43(5):1367-1389

Schuknecht HF, Watanuki K, Takahashi T, Belal AA Jr, Kimura RS, Jones DD, Ota CY (1974) Atrophy of the stria vascularis, a common cause for hearing loss. Laryngoscope 84(10):17771821

Shannon RV (1976) Two-tone unmasking and suppression in a forward-masking situation. J Acoust Soc Am 59(6):1460-1470

Sommers MS, Gehr SE (1998) Auditory suppression and frequency selectivity in older and younger adults. J Acoust Soc Am 103(2):1067-1074

Sommers MS, Gehr SE (2010) Two-tone auditory suppression in younger and older normal-hearing adults and its relationship to speech perception in noise. Hear Res 264:56-62

Winslow RL, Sachs MB (1987) Effect of electrical stimulation of the crossed olivocochlear bundle on auditory nerve response to tones in noise. J Neurophysiol 57(4):1002-1021

Yasin I, Drga V, Plack CJ (2014) Effect of human auditory efferent feedback on cochlear gain and compression. J Neurosci 34(46):15319-15326 